\title{
Decreased activity of desaturase 5 in association with obesity and insulin resistance aggravates declining long-chain $n-3$ fatty acid status in Cree undergoing dietary transition
}

\author{
Yuan E. Zhou ${ }^{1}$, Stan Kubow ${ }^{1}$, Eric Dewailly ${ }^{2}$, Pierre Julien ${ }^{2}$ and Grace M. Egeland ${ }^{1 *}$ \\ ${ }^{1}$ School of Dietetics and Human Nutrition, McGill University, CINE Building, 21,111 Lakeshore Road, Ste Anne de Bellevue, \\ H9X 3V9 Montreal, Canada \\ ${ }^{2}$ Unité de Recherche en Santé Publique, Centre de Recherche du CHUQ, Universite Laval, G1V 2M2 Quebec, Canada \\ (Received 29 August 2008 - Revised 27 January 2009 - Accepted 6 February 2009 - First published online 2 April 2009)
}

Emerging evidence shows that desaturase $5(\Delta 5)$, the key regulator in the synthesis of highly unsaturated long-chain fatty acids (HUFA), is modulated by factors including adiposity, diet and insulin resistance. We explored the association of these factors in a cross-sectional study within a high-risk Cree population. Anthropometric measures and fasting blood glucose and insulin were analysed. $\Delta 5$ was estimated as the $20: 4 n$ 6:20:3n-6 ratio in erythrocyte membranes. The setting of the present study was the Mistissini community in the Cree Territory of Québec, Canada with ninety-eight female and sixty-eight male subjects aged $20-88$ years. Obesity (BMI $\geq 30 \mathrm{~kg} / \mathrm{m}^{2}$ ) was prevalent across age groups. $\Delta 5$ was inversely associated with BMI (Spearman's correlation coefficient $\left.\left(r_{\mathrm{s}}\right)-0.175 ; P=0.03\right)$ and positively associated with age $\left(r_{\mathrm{s}} 0.593\right.$; $P<0.0001)$, which was driven by age-related increases in dietary intake of $n$ - 3 fatty acids and decreases in $20: 3 n-6$. Homeostasis model assessment of insulin resistance (HOMA-IR) was significantly inversely associated with $\Delta 5$ in age-adjusted linear regression analyses in normoglycaemic individuals $(\beta-2.110$ (SE 0.566); $P<0.001$ ), whereas no association was observed among glucose-intolerant individuals (interaction term $P=0.03$ ). In contrast, there were no significant interactions indicating differences in the slope for each of the adiposity measures in their associations with $\Delta 5$. The present study indicates that the dietary transition of reduced consumption of fish among younger Cree may compound the effects of obesity and emerging insulin resistance which, in turn, could reduce bioavailability of HUFA $n$ - 3 (through reduced $\Delta 5$ activity). Also, the study suggests that disease progression is an important consideration when evaluating correlates of $\Delta 5$ activity in observational studies.

Desaturase 5: Obesity: Insulin resistance: Indigenous peoples: Dietary transition

Consequences of obesity include insulin resistance, dyslipidaemia, hypertension and the development of overt diabetes and CVD with reduced life expectancy ${ }^{(1)}$. There are multiple obesity-mediated pathways that are known and/or suspected to contribute to the influence of adiposity on metabolic disturbances such as excessive NEFA released from adipose tissue and its inhibitory effect on tissue insulin sensitivity, or a low-grade inflammation related to obesity ${ }^{(2)}$. Fatty acids, particularly highly unsaturated fatty acids (HUFA), are considered as important aetiological factors for a variety of obesity-related illnesses. HUFA are important for the maintenance of normal biomembrane structure and function ${ }^{(3)}$ and are precursors of eicosanoids that have important physiological properties affecting numerous cardiovascular, immune and cellular secretory functions ${ }^{(4-7)}$. Additionally, HUFA are key regulators of many genes involved in controlling lipid homeostasis and may thereby help to lessen dyslipidae$\mathrm{mia}^{(3)}$. Further, the anti-arrhythmic action of $n-3$ HUFA is considered to be protective against myocardial infarction and IHD via increasing cell membrane fluidity ${ }^{(5)}$, influencing membrane ion channel function and regulating cytosolic sodium ion and L-type $\mathrm{Ca}$ ion levels in cardiac myocytes ${ }^{(5,6)}$.
As HUFA are derived from both exogenous and endogenous sources, both dietary intake of fatty acids and factors influencing endogenous synthesis could contribute to disease risk. Desaturase $5(\Delta 5)$ is the key enzyme in the endogenesis pathways of $n-3$ and $n-6$ HUFA. Although there is no direct measurement of $\Delta 5$, its activity can be estimated by the ratio of precursor fatty acid to the subsequent 'daughter' fatty acids present in serum ${ }^{(8,9)}$, plasma ${ }^{(10)}$, erythrocytes ${ }^{(11)}$ and skeletal muscle membranes ${ }^{(12)}$. In that regard, the plasma $20: 4 n-6: 20: 3 n-6$ ratio in human studies is typically used as a surrogate measure of $\Delta 5$ activity. The $20: 5 n-3: 20: 4 n-3$ ratio is also reflective of enzymic $\Delta 5$ activity; however, this latter ratio is not used in human studies due to the low or undetectable amounts of $20: 4 n-3$ in human plasma.

Indigenous peoples residing in the Canadian North have a paradoxical coexistence of obesity with low CVD risk $^{(13)}$, which has been related to a relatively high intake of $n-3$ fatty acids from traditional foods. Foods that are traditionally consumed by Cree include lake trout, whitefish, burbot, walleye and sturgeon, which contain significant amount of DHA and $\mathrm{EPA}^{(14-16)}$. Also, Cree typically consume wild

Abbreviations: $\Delta 5$, desaturase 5; HOMA-IR, homeostasis model assessment of insulin resistance; HUFA, highly unsaturated long-chain fatty acids. 
fish, which has higher levels of $n-3$ fatty acids than fish from aquaculture sources ${ }^{(17)}$. On the other hand, indigenous peoples are undergoing rapid dietary transition that could diminish $n-3$ HUFA intake leading to increased chronic disease $\operatorname{risk}^{(18,19)}$. Thus, assessment of plasma HUFA status among indigenous peoples undergoing dietary transition is important. The present study has focused upon the Cree of James Bay as they have continued to undergo dietary transition away from traditional food rich in $n-3$ fatty acids ${ }^{(20)}$.

Among the endogenous regulatory factors of $\Delta 5$, insulin is of particular interest as its administration in a wide age range of healthy subjects increases activity of $\Delta 5^{(10)}$. Also, an inverse association has been observed between insulin resistance levels and $\Delta 5$ activity in cross-sectional studies of apparently healthy individuals ${ }^{(8,12)}$ and in a 20 -year prospective follow-up study of middle-aged men ${ }^{(9)}$. The data from these latter studies suggest that, in addition to dietary intake of HUFA, altered insulin resistance levels can modify HUFA tissue profile via the regulation of $\Delta 5$ activity. Independent of insulin action, adiposity could also impact upon $\Delta 5$ activity and tissue HUFA profiles, as clinical studies have demonstrated strong negative correlations between adiposity measures and $\Delta 5$ activity ${ }^{(8,9,12)}$. In that regard, obese Zucker rats demonstrated reduced affinity of hepatic $\Delta 5$ to its substrates as compared with their lean rat counterparts, which was not explainable by differences in insulinaemia ${ }^{(21)}$.

In the present study, we explored the associations of $\Delta 5$ activity and erythrocyte levels of HUFA with measures of adiposity and insulin resistance in a James Bay Cree population undergoing dietary transition away from traditional food.

\section{Methods}

\section{Location and subjects}

A cross-sectional study was conducted in the community of Mistissini, Québec in the summer of 2005 and was coordinated by the Cree Board of Health in collaboration with McMaster, Laval and McGill universities. The community survey was the first in an ongoing study entitled 'Nituuchischaayihitaau Aschii: A Multi-Community Environment-and-Health Longitudinal Study in Iiyiyiu Aschii'. Background information on selected aspects of the methodology and study population is provided elsewhere ${ }^{(22)}$. Briefly speaking, local publicity were employed to raise public awareness on the project. A stratified random sampling consisting of four age strata was used to select the residents from a municipal list of the community of Mistissini. Pregnant women were excluded. The recruitment and interviews were conducted with assistance from qualified local bilingual Iiyiyiuch interviewers. Among 359 adults (age $\geq 20$ years) who were randomly selected, seventy-nine of them were out of town or not able to be contacted. For the 280 potential participants, $62 \%$ of them participated in the study ( $n$ 172). Ethics approval was obtained from participating institutions. While the community study included children, the current analyses were restricted to adults.

Among the 172 adult participants, fasting blood samples were available from 166 individuals (ninety-eight women and sixty-eight men) aged 20-88 years for the measurement of erythrocyte membrane fatty acids, serum glucose and insulin levels. Weight and body fat percentage were measured using a bioelectrical impedance scale (Tanita, Tokyo, Japan). Height was measured without shoes to the nearest $\mathrm{cm}$ using a stadiometer with the patient standing on a hard surface. BMI was calculated $\left(\mathrm{kg} / \mathrm{m}^{2}\right)$. Waist circumference was measured at the end of exhalation with the tape placed horizontally between the last floating rib and the iliac crest. Obesity was defined by BMI $\geq 30 \mathrm{~kg} / \mathrm{m}^{2}$ and abdominal obesity was defined by the waist (waist $\geq 102 \mathrm{~cm}$ in men and $\geq 88 \mathrm{~cm}$ in women) ${ }^{(23)}$.

\section{Laboratory analyses}

Fatty acids. Blood concentrations of fatty acids were determined in erythrocyte membranes by GLC. Erythrocytes $(300 \mu \mathrm{l})$ were thawed and lysed in $1 \mathrm{ml}$ water. Membranes were isolated by centrifugation $(21000 \mathrm{~g}$ for $15 \mathrm{~min})$ and washed twice with a $0.9 \% \mathrm{NaCl}$ solution. The pellet was spiked with an internal standard of phosphatidylcholine 15:0 (Avanti Polar Lipids, Alabaster, AL, USA) and lipids were liquid-liquid extracted using chloroform and methanol (2:1, $\mathrm{v} / \mathrm{v}$ ) according to a modified Folch method ${ }^{(24)}$. Fatty acids from membrane phospholipids were methylated in methanolbenzene $(4: 1, \mathrm{v} / \mathrm{v})$ mixed with acetyl chloride according to previously described methods ${ }^{(25)}$. Fatty acid profiles were obtained by capillary GC using a temperature gradient on a HP5890 gas chromatograph (Hewlett Packard, Toronto, Canada) equipped with an HP8823 capillary column coupled with a flame ionisation detector. The elution gas used was He (split ratio 1:72). Fatty acids were identified according to their retention time on the column, using a standard mixture of thirty-seven fatty acids as a basis for comparison (FAME $37 \mathrm{mix}$; Supelco Inc., Bellefonte, PA, USA), which contained the fatty acid standard $15: 0$, as well as a mixture of thirty-one fatty acids (GLC-411; NuCheck Prep Inc., Elysian, MN, USA) and a mixture PUFA-3 (Matreya Inc., Ontario, Canada). Results were expressed as percentage of total fatty acids.

Blood glucose and insulin. Plasma glucose was measured enzymically, and fasting insulin concentrations were measured with a commercial double-antibody RIA as described by Dewailly et al. ${ }^{(17)}$.

\section{Statistical analysis}

Data for continuous variables are presented as mean values and standard deviations if normally distributed and as the geometric mean with $95 \%$ CI if not normally distributed. Categorical variables were calculated as proportions. Fatty acid content in erythrocyte membranes was expressed as a percentage of total fatty acids. SFA, MUFA, $n-3$ PUFA, $n$-6 PUFA and trans-fatty acids were calculated by summing the concentrations of individual acids if they were detectable. The activity of $\Delta 5$ was estimated by the $20: 4 n-6: 20: 3 n-6$ ratio. Insulin resistance was estimated by the homeostasis model assessment of insulin resistance (HOMA-IR), calculated as follows:

$$
\mathrm{HOMA}-\mathrm{IR}=(\text { fasting insulin }(\mu \mathrm{U} / \mathrm{ml})
$$

$\times$ fasting plasma glucose $(\mathrm{mmol} / 1)) / 22 \cdot 5$. 
As the age span of the present study population ranged from 20 to 88 years, subjects were divided into four age groups. Linear regression was applied to detect linear trends of the anthropometric measures, HOMA-IR, fatty acid profiles of erythrocyte membranes and $\Delta 5$ activity across age groups. Interactions between HOMA-IR and anthropometric measures were tested with general linear models. The general linear model was conducted to test linear trends of continuous variables across age groups. The generalised regression model was used to test linear trends of categorical variables across age groups. As there were no observed interactions of sex on $\Delta 5$-related associations, the results of associations of $\Delta 5$ with anthropometric measures and HOMA-IR were presented in a combined manner for all subjects. Spearman correlations were conducted for the correlations of $\Delta 5$ with adiposity measures, HOMA-IR and age. Age-adjusted multivariable linear regression was used to: (1) separately evaluate the associations of $\Delta 5$ with HOMA-IR; (2) separately assess the associations of $\Delta 5$ with BMI, body fat percentage and waist circumference. Because disease state may influence the association of HOMA-IR with $\Delta 5$, the interaction term was included in the age-adjusted linear regression analyses to test the interaction of fasting glucose status on the association between HOMA-IR and desaturase $\Delta 5$. All $P$ values were obtained from two-sided tests. Data were analysed with SAS software (version 9.1; SAS Institute Inc., Cary, NC, USA).

\section{Results}

\section{Subject characteristics}

The present study population was composed of ninety-eight females (aged 40.1 (SD 15.8) years; BMI $34.9($ SD 6.8$) \mathrm{kg} / \mathrm{m}^{2}$ ) and sixty-eight males (mean age 40.7 (SD 16.1) years; mean BMI $31.5(\mathrm{SD} 5.5) \mathrm{kg} / \mathrm{m}^{2}$ ). There were no significant differences among age groups in adiposity measures ( $P$ for trend NS) (Table 1). The geometric mean HOMA-IR increased slightly and non-significantly $(P<0 \cdot 1)$ with increasing age, whereas the proportion of subjects with impaired fasting glucose (i.e. fasting glucose defined as $\geq 5.7 \mathrm{mmol} / \mathrm{l})^{(26)}$ increased significantly with advancing age $(P<0 \cdot 0001)$.

\section{Erythrocyte fatty acid content}

Fatty acid classes of erythrocyte membranes showed distinctive associations with age (Table 2). In particular, total $n-3$ fatty acids increased significantly across age groups $(P<0.0001)$. The latter observed association was driven mainly by two HUFA fatty acids, $20: 5 n-3$ and $22: 6 n-3$, which consistently and significantly increased with age $(P<0 \cdot 0001)$. Conversely, total $n-6$ fatty acids decreased significantly across age groups $(P<0 \cdot 0001)$. Almost all individual $n-6$ fatty acids showed the same strong and inverse associations with age $(P<0 \cdot 0001)$ except for $20: 4 n-6$ whose percentage in the fatty acid profile of erythrocyte membranes differed only slightly between different age groups $(P=0 \cdot 05)$.

As expected, $n-3$ fatty acids were inversely correlated with $n$-6 fatty acids $\left(r_{\mathrm{s}}-0.716 ; P \leq 0 \cdot 0001\right)$. Similarly, $20: 3 n-6$ negatively correlated with $20: 5 n-3(r-0.463 ; P<0.0001)$ and $22: 6 n-3(r-0.503 ; P<0.0001)$. In terms of the numerator and denominator of the ratio representing $\Delta 5$ activity, the numerator $(20: 4 n-6)$ increased with age $(P=0.05)$ while the denominator $(20: 3 n-6)$ decreased with age $(P<0 \cdot 0001)$, resulting in significant increases in $\Delta 5$ across all age groups $(P<0.0001)$ (Table 2).

The proportion of total SFA increased with age $(P<0.05)$, but the increase was subtle and there was no significant difference in individual SFA level by age. In terms of other fatty acid classes, i.e. MUFA and trans-fatty acids, no significant differences were observed across age groups for either the total fatty acid class or for any individual fatty acid.

\section{Desaturase 5, homeostasis model assessment of insulin resistance and adiposity}

Negative correlations were observed between $\Delta 5$ and adiposity measures, particularly BMI $\left(r_{\mathrm{s}}-0.175 ; P<0.05\right)$ and

Table 1. Clinical characteristics of the study population

(Mean values and standard deviations)

\begin{tabular}{|c|c|c|c|c|c|c|c|c|c|}
\hline \multirow[t]{2}{*}{ Age (years)... } & \multicolumn{2}{|c|}{$20-29$} & \multicolumn{2}{|c|}{$30-39$} & \multicolumn{2}{|c|}{$40-49$} & \multicolumn{2}{|c|}{$50+$} & \multirow[b]{2}{*}{$P$ for trend } \\
\hline & Mean & SD & Mean & SD & Mean & SD & Mean & SD & \\
\hline$n$ & \multicolumn{2}{|c|}{51} & \multicolumn{2}{|c|}{46} & \multicolumn{2}{|c|}{30} & \multicolumn{2}{|c|}{39} & \\
\hline BMI $\left(\mathrm{kg} / \mathrm{m}^{2}\right)^{\star}$ & $33 \cdot 1$ & $7 \cdot 3$ & $34 \cdot 1$ & $6 \cdot 7$ & 33.8 & $5 \cdot 0$ & $33 \cdot 3$ & $6 \cdot 5$ & NS \\
\hline Body fat $(\%)^{\star}$ & $38 \cdot 7$ & 11.5 & 39.9 & $9 \cdot 4$ & $40 \cdot 4$ & $8 \cdot 4$ & $40 \cdot 5$ & $8 \cdot 0$ & NS \\
\hline Waist girth $(\mathrm{cm}) \dagger$ & $107 \cdot 0$ & $15 \cdot 7$ & $110 \cdot 2$ & 14.4 & $109 \cdot 3$ & $11 \cdot 0$ & $111 \cdot 2$ & 14.7 & NS \\
\hline Proportion of obesity by BMI (\%)‡ & \multicolumn{2}{|c|}{$58 \cdot 3$} & \multicolumn{2}{|c|}{$71 \cdot 1$} & \multicolumn{2}{|c|}{$82 \cdot 8$} & $62 \cdot 9$ & & NS \\
\hline Proportion of obesity by waist girth (\%)§ & & & \multicolumn{2}{|c|}{$82 \cdot 2$} & \multicolumn{2}{|c|}{$79 \cdot 3$} & \multicolumn{2}{|c|}{89.7} & NS \\
\hline Proportion of females (\%) & \multicolumn{2}{|c|}{$\begin{array}{l}72 \cdot 9 \\
56 \cdot 9\end{array}$} & & & & & \multicolumn{2}{|c|}{$59 \cdot 0$} & NS \\
\hline HOMA-IR & & & & & & & & & $<0.1$ \\
\hline Geometric mean & \multicolumn{2}{|c|}{$5 \cdot 6$} & \multicolumn{2}{|c|}{$6 \cdot 4$} & \multicolumn{2}{|c|}{$6 \cdot 7$} & \multicolumn{2}{|c|}{$7 \cdot 6$} & \\
\hline $95 \% \mathrm{Cl}$ & \multicolumn{2}{|c|}{$\begin{array}{c}4 \cdot 7,6 \cdot 8 \\
25.5\end{array}$} & \multirow{2}{*}{\multicolumn{2}{|c|}{$\begin{array}{c}5 \cdot 1,7 \cdot 9 \\
47.8\end{array}$}} & \multicolumn{2}{|c|}{$\begin{array}{c}5 \cdot 1,8.7 \\
66.7\end{array}$} & \multicolumn{2}{|c|}{$5 \cdot 9,9 \cdot 7$} & \\
\hline Proportion of impaired fasting glucose (\%) & \multicolumn{2}{|c|}{$25 \cdot 5$} & & & \multicolumn{2}{|c|}{$66 \cdot 7$} & \multicolumn{2}{|c|}{$82 \cdot 1$} & $<0.0001$ \\
\hline
\end{tabular}

HOMA-IR, homeostasis model assessment of insulin resistance.

${ }^{*}$ Age group 18-29 years, $n$ 48; age group 30-39 years, $n$ 45; age group 40-49 years, $n$ 29; age group $50+$ years, $n 35$.

† Age group 18-29 years, $n$ 48; age group 30-39 years, $n 45$; age group 40-49 years, $n 29$; age group $50+$ years, $n 39$.

‡Proportion of subjects with BMI $\geq 30 \mathrm{~kg} / \mathrm{m}^{2}$.

$\S$ Proportion of subjects with waist girth $\geq 88 \mathrm{~cm}$ (female) or $\geq 102 \mathrm{~cm}$ (male). 
Table 2. Proportion (\% of total fatty acids) of fatty acids in erythrocytes of the study population (Mean values and standard deviations)

\begin{tabular}{|c|c|c|c|c|c|c|c|c|c|}
\hline \multirow[t]{2}{*}{ Age (years)... } & \multicolumn{2}{|c|}{$20-29$} & \multicolumn{2}{|c|}{$30-39$} & \multicolumn{2}{|c|}{$40-49$} & \multicolumn{2}{|c|}{$50+$} & \multirow[b]{2}{*}{$P$ for trend } \\
\hline & Mean & SD & Mean & SD & Mean & SD & Mean & SD & \\
\hline$n$ & \multicolumn{2}{|c|}{51} & \multicolumn{2}{|c|}{46} & \multicolumn{2}{|c|}{30} & \multicolumn{2}{|c|}{39} & \\
\hline SFA & 41.89 & 0.74 & 41.83 & 0.77 & $42 \cdot 29$ & 0.74 & $42 \cdot 31$ & 0.85 & $<0.05$ \\
\hline $14: 0$ & 0.31 & 0.04 & 0.32 & 0.04 & 0.32 & 0.04 & 0.29 & 0.07 & NS \\
\hline $16: 0$ & $19 \cdot 66$ & 0.77 & 19.82 & 0.61 & $19 \cdot 76$ & 0.71 & $19 \cdot 88$ & 0.70 & NS \\
\hline $18: 0$ & 14.75 & 0.46 & $14 \cdot 76$ & 0.49 & $14 \cdot 88$ & 0.70 & 14.78 & 0.62 & NS \\
\hline MUFA & $18 \cdot 79$ & 0.84 & $18 \cdot 75$ & 0.93 & $18 \cdot 36$ & 0.62 & $18 \cdot 77$ & $1 \cdot 18$ & NS \\
\hline $16: 1 n-7$ & 0.32 & 0.20 & 0.37 & 0.15 & 0.32 & 0.16 & 0.29 & 0.27 & NS \\
\hline $18: 1 n-9$ & 11.40 & 0.60 & 11.40 & 0.77 & $11 \cdot 29$ & 0.69 & 11.74 & 1.04 & NS \\
\hline $18: 1 n-7$ & 1.46 & 0.35 & 1.50 & 0.35 & 1.30 & 0.36 & 1.30 & 0.36 & NS* \\
\hline $24: 1 n-9$ & 4.96 & 0.40 & 4.84 & 0.31 & 4.84 & 0.40 & 4.89 & 0.47 & NS \\
\hline n-6 PUFA & 31.96 & 0.80 & 31.47 & 0.92 & $31 \cdot 14$ & 0.87 & 29.43 & 1.60 & $<0.0001$ \\
\hline$n-6$ HUFA & $18 \cdot 60$ & 0.88 & $18 \cdot 65$ & 0.89 & 18.63 & 0.68 & $18 \cdot 23$ & $1 \cdot 14$ & 0.05 \\
\hline $18: 2 n-6$ & 10.94 & 0.65 & 10.51 & 0.69 & $10 \cdot 40$ & 0.78 & 9.50 & $1 \cdot 15$ & $<0.0001$ \\
\hline $20: 3 n-6$ & $2 \cdot 12$ & 0.29 & 2.00 & 0.27 & 1.86 & 0.31 & 1.46 & 0.34 & $<0.0001$ \\
\hline $20: 4 n-6$ & $14 \cdot 32$ & 0.74 & 14.54 & 0.67 & 14.59 & 0.49 & 14.61 & 0.84 & 0.05 \\
\hline $22: 4 n-6$ & 3.39 & 0.40 & 3.24 & 0.34 & $3 \cdot 17$ & 0.35 & 2.85 & 0.37 & $<0.0001$ \\
\hline $22: 5 n-6$ & 0.89 & 0.12 & 0.87 & $0 \cdot 13$ & 0.88 & 0.10 & 0.77 & 0.17 & $<0.0001$ \\
\hline n-3 PUFA & 5.89 & 0.70 & 6.47 & 0.69 & $6 \cdot 84$ & 0.64 & $8 \cdot 11$ & 1.39 & $<0.0001$ \\
\hline n-3 HUFA & 5.40 & 0.63 & 5.88 & 0.62 & $6 \cdot 38$ & 0.66 & $7 \cdot 70$ & 1.31 & $<0.0001$ \\
\hline $18: 3 n-3$ & 0.15 & 0.10 & 0.15 & 0.11 & 0.15 & 0.10 & 0.13 & 0.16 & NS \\
\hline $20: 5 n-3$ & 0.37 & 0.09 & 0.43 & 0.11 & 0.47 & 0.11 & 0.73 & 0.29 & $<0.0001$ \\
\hline $22: 3 n-3$ & 0.20 & 0.15 & 0.24 & $0 \cdot 13$ & 0.18 & 0.15 & $0 \cdot 15$ & 0.17 & NS \\
\hline $22: 6 n-3$ & 2.97 & 0.54 & 3.44 & 0.54 & $3 \cdot 82$ & 0.62 & $4 \cdot 80$ & 1.03 & $<0.0001$ \\
\hline$\Delta 5 \dagger$ & 6.89 & 1.08 & 7.43 & 1.22 & 8.06 & 1.45 & $10 \cdot 60$ & $2 \cdot 62$ & $<0.0001$ \\
\hline
\end{tabular}

HUFA, highly unsaturated long-chain fatty acids; $\Delta 5$, desaturase 5 .

${ }^{*}$ No clinical significance, because although $P<0.01$ for the general linear model, $R^{2}$ is only $4 \%$ and $\beta$ is too small $(-0.005)$. $\dagger \Delta 5$ is estimated as the $20: 4 n-6: 20: 3 n-6$ ratio.

percentage body fat $\left(r_{\mathrm{s}}-0.168 ; P<0.05\right)$. The activity of $\Delta 5$ was not correlated significantly with HOMA-IR in the unadjusted analyses (Table 3). Upon further analyses of fasting glucose status, however, a significant interaction term showed differences in the slope of HOMA-IR with $\Delta 5$ as the dependent variable $(P=0.03)$. In that regard, a strong and significant inverse relationship of HOMA-IR with $\Delta 5$ was observed among those with normal fasting glucose but not those with impaired fasting glucose. There was no observed significant interaction of fasting glucose status on the associations between adiposity and $\Delta 5$. Thus, all the subjects were combined together in separate age-adjusted models evaluating BMI, percentage body fat and waist circumference for their associations with $\Delta 5$. The adjusted model using $\Delta 5$ as the dependent variable showed for BMI: $\beta-0.037$, SE 0.019 , $P=0.05$; for percentage body fat: $\beta-0.041$, SE 0.013 , $P=0.002$; and for waist: $\beta-0.020$, SE $0.009, P=0.03$ (Table 4).

\section{Discussion}

In the present study, HUFA $n-3$ fatty acids, especially $22: 6 n-3$, increased significantly with age, while $n-6$ fatty acids showed an opposite trend. These latter findings are consistent with previous observations that older Cree consume more traditional food rich in HUFA $n-3$ fatty acids, while young Cree consume more market foods that are rich in $n-6$ fatty acids ${ }^{(20)}$. In that regard, plasma EPA and DHA were shown to increase significantly with age in an earlier study of James Bay Cree ${ }^{(2)}$. High consumption of traditional food, including fish, has been reported among older community members throughout northern indigenous communities in the three Canadian territories ${ }^{(27)}$. In the present study, 20:3n-6 negatively correlated with the two $n-3$ fatty acids, $20: 5 n-3$ and $22: 6 n-3$, that are abundant in fish and sea food $^{(28,29)}$. As only trace amounts of $20: 3 n-6$ are present in food, this fatty acid is primarily indicative of endogenous metabolic activity. Since $n-3$ fatty acids and $n-6$ fatty acids compete

Table 3. Spearman correlation coefficients among adiposity, homeostasis model assessment of insulin resistance (HOMA-IR) and age

\begin{tabular}{|c|c|c|c|c|c|}
\hline & Body fat percentage & BMI & Waist & HOMA-IR & Age \\
\hline BMI & $0.777^{\star \star \star \star}$ & & & & \\
\hline Waist & $0.650^{\star \star \star \star}$ & $0.907^{\star \star \star \star}$ & & & \\
\hline HOMA-IR & $0.557^{\star \star \star *}$ & $0.599^{\star \star \star *}$ & $0.568^{\star \star \star \star}$ & & \\
\hline Age & 0.013 & 0.012 & 0.073 & $0.145 \dagger$ & \\
\hline$\Delta 5$ & $-0.168^{*}$ & $-0.175^{\star}$ & -0.116 & -0.112 & $0.593^{\star * * *}$ \\
\hline
\end{tabular}

$\triangle 5$, desaturase 5 .

${ }^{\star} P<0.05,{ }^{* \star} P<0.01,{ }^{\star \star * \star} P<0.001,{ }^{* \star \star \star} P<0.0001$.

$\dagger 0.1<P<0.05$. 
Table 4. Age-adjusted regression coefficients of adiposity measures and homeostasis model assessment of insulin resistance (HOMA-IR) and their individual associations with desaturase $5^{*}$

( $\beta$ Coefficients with their standard errors)

\begin{tabular}{lcccc}
\hline Independent variables & $\beta$ & SE & $P$ & $R_{\text {adj }}^{2} \dagger$ \\
\hline Body fat percentage $(n$ 157) & -0.041 & 0.013 & 0.002 & 0.515 \\
BMI $(n$ 157) & -0.037 & 0.019 & 0.05 & 0.496 \\
Waist $(n$ 161) & -0.020 & 0.009 & 0.03 & 0.479 \\
HOMA-IR & & & & \\
Fasting glucose $<5.7 \mathrm{mmol} / \mathrm{l}(n 79)$ & -2.110 & 0.566 & $<0.001$ & 0.511 \\
Fasting glucose $\geq 5.7 \mathrm{mmol} / \mathrm{l} 87)$ & -1.015 & 0.674 & 0.14 & 0.419 \\
\hline
\end{tabular}

${ }^{*}$ Adjusted for age and separately assessed.

$\dagger R_{\text {adj }}^{2}$ refers to the whole model.

with each other within membrane phospholipids ${ }^{(30)}$ and for the desaturases $^{(31)}$, increased intake of fatty acids from one class leads to the reduced presence of fatty acids in membranes from the other classes. Hence, a reasonable explanation is that higher consumption of fish in older Cree inhibits elongation and desaturation of $n-6$ fatty acids in these subjects, leading to the negative relationship between $n$-3 HUFA and 20:3n-6 observed in the erythrocyte membrane fatty acid profiles.

The fatty acid composition of erythrocyte membranes reflects the dietary intake of fatty acids which are mainly exogenously originated, such as HUFA, trans-fatty acids and odd-number fatty acids. Although the fatty acid profile of erythrocyte membranes is modulated by both endogenous pathways and dietary intake, the efficiency of endogenous synthetic pathways of $n-3$ HUFA is recognised to be rather low. Based on clinical trials using isotopically labelled tracer fatty acids or analysing blood fatty acid profile after dietary supplementation, a recent review concluded that only about $5 \%$ of $18: 3 n-3$ consumed is transformed to $20: 5 n-3$ and less than $0.5 \%$ of $18: 3 n-3$ is converted into $22: 6 n-3^{(32)}$. Hence, the erythrocyte membrane $n-3$ HUFA content reflects primarily dietary intake. The measurement of fatty acid profiles of erythrocyte membranes used in the present study has been extensively tested in dietary PUFA intervention studies using dietary sources of $20: 5 n-3$ and $22: 6 n-3^{(33,34)}$ and is commonly used as an evaluation of HUFA intake in epidemiological studies ${ }^{(35)}$. Normally the endogenous pathway of HUFA biosynthesis exerts a relatively minor influence on the HUFA content of erythrocyte membranes. When dietary HUFA intake is low, however, the contribution of endogenous HUFA biosynthesis towards erythrocyte HUFA content becomes physiologically significant. Since dietary fatty acid intake was closely related to age in the present study, an adjustment for age was performed to eliminate the confounding influence of fatty acid intake on $\Delta 5$ activity, which was estimated by the $20: 4 n-6: 20: 3 n-6$ ratio in erythrocyte membranes. Thus, the impact of other metabolic influences on $\Delta 5$ activity, such as obesity and insulin resistance, was assessed by adjusting for the confounding effects of age and dietary fatty acid intake.

Interestingly, in the present study population, there was no significant difference of obesity prevalence between age groups in both sexes. Obesity was prevalent in the study population as shown by the mean BMI of $>33 \mathrm{~kg} / \mathrm{m}^{2}$, including more than one-third of subjects having BMI of $\geq 35 \mathrm{~kg} / \mathrm{m}^{2}$ (data not shown) and a mean waist circumstance $>106 \mathrm{~cm}$ in all of the age groups. Both prevalence of obesity and the average BMI level among all age groups were much higher than previously reported in a recent Canadian national survey of the non-indigenous population ${ }^{(36)}$ or data from an earlier national survey of multiple ethnic populations in the $\mathrm{USA}^{(37)}$.

A significant proportion of Cree subjects had impaired fasting glucose level $(\geq 5.7 \mathrm{mmol} / \mathrm{l})$ and this proportion increased with age. At normal fasting glucose conditions $(<5.7 \mathrm{mmol} / \mathrm{l})$, a positive association exists between levels of insulin and glucose ( $r$ 0.417, $P=0.0001$; data not shown) among the Cree. This correlation, however, disappeared in the subgroup with impaired fasting glucose, indicating that insulin's regulation was disrupted. Hence, it was not surprising that an inverse association between HOMA-IR and $\Delta 5$ was observed among subjects with normal fasting glucose, while this association became non-significant among subjects with impaired fasting glucose.

In contrast, no significant interaction was observed for the effects of adiposity on $\Delta 5$ between normoglycaemic and impaired glucose-tolerant individual, suggesting that gradients of adiposity continue to play an important role in $\Delta 5$ activity regardless of the degree of disease progression. In the present study, body fat percentage was a more significant negative predictor of $\Delta 5$ than waist girth, which may be related to the unusually high adiposity of the Cree study population. In the presence of high adiposity, effects of abdominal adiposity on metabolic consequences may be diluted, since Health Canada suggests that waist girth is not to be considered as an additive risk for individuals with $\mathrm{BMI} \geq 35 \mathrm{~kg} / \mathrm{m}^{2(23)}$. Similar to the Cree, inverse associations between adiposity level and $\Delta 5$ have been reported in several studies including Pima Indians $^{(12)}$, obese Hungarian children ${ }^{(38)}$ and Caucasians ${ }^{(8)}$. Among Pima Indians, the $20: 4 n-6: 20: 3 n-6$ ratio in skeletal muscle membranes was significantly and strongly inversely related to adiposity measures ${ }^{(12)}$. A study on Hungarian children showed that the $20: 4 n-6: 20: 3 n-6$ ratio in plasma phospholipids was reduced in obese children compared with normal controls and this ratio was lowest among obese subjects with the metabolic syndrome ${ }^{(38)}$. The latter observation may suggest that obesity and other metabolic syndrome components such as insulin resistance may have additive effects on suppressing $\Delta 5$ activity. A recent study on apparently healthy middle-aged Caucasians reported a negative correlation between the $20: 4 n-6: 20: 3 n-6$ ratio in serum cholesteryl esters and sagittal abdominal diameter that was independent 
of age ${ }^{(8)}$. Evidence from in vitro studies and rodent models also suggests a direct link between adiposity and $\Delta 5$ activity. In one in vitro study, fresh microsomes taken from obese and lean Zucker rats were incubated with the substrate $20: 3 n-6$ at physiological concentrations. $\Delta 5$ activity, measured as $20: 4 n$ 6 formed per time unit per weight unit of liver microsomal protein, was lower in freshly isolated microsomes from obese Zucker rats at all age stages, which correlated with in vivo observations of $\Delta 5$ activity (as assessed by hepatic $20: 4 n-6: 20: 3 n-6$ ratios) being lower in obese than in lean Zucker rats ${ }^{(21)}$. The lower $\Delta 5$ activity in the hepatic microsomes in the obese Zucker rats was not accountable for the hyperinsulinaemic condition of the obese Zucker rats. Similar findings have been reported ${ }^{(39)}$ in some but not all ${ }^{(40)}$ studies involving $o b / o b$ mice. In the present work, no significant interaction was observed for the effects of adiposity on $\Delta 5$ between normoglycaemic and impaired fasting glucose individuals, suggesting that gradients of adiposity continue to play an important modulatory role in $\Delta 5$ activity regardless of the degree of disease progression involving glucose intolerance.

In most populations, advancing age typically leads to increasing adiposity and reduced insulin sensitivity with a consequent decrease in $\Delta 5$ activity. Among the Cree, however, adiposity was not related to age and age was only weakly and non-significantly related to insulin resistance. Therefore, age cannot account for the observed relationships of the adiposity measures with $\Delta 5$ seen among the studied population of Cree subjects or the observed association between HOMA-IR with $\Delta 5$ observed among Cree subjects with normal fasting glucose. Additionally, the statistical adjustment for age used in the present study adjusts for the observed age-related differences in dietary intake. To date, animal studies show inconsistent findings regarding the importance of age on $\Delta 5$ activity $^{(41,42)}$, indicating that future studies are warranted in this regard.

Several limitations should be recognised in the present study. The causal relationships between the variables of interest cannot be determined based on the cross-sectional data. In addition, more precise measurements of body composition such as dualenergy X-ray absorptiometry could be considered to detect subcutaneous adipose tissue and visceral adipose tissue in future studies, which would clarify which part of adipose tissue has more significant influence on fatty acid metabolism. Future studies are needed to observe whether the results from the current studies can be generalised to other populations.

The results reported herein indicate that the high prevalence of obesity among Cree of James Bay concomitant with a low $n$-3 HUFA intake could exacerbate chronic disease risks through reduction in $\Delta 5$ activity. The activity of $\Delta 5$ may be important for disease prevention, as this enzyme is critical for the synthesis of optimal $n-3$ HUFA tissue profiles that are associated with anti-inflammatory and anti-arrhythmic cardioprotective effects. These findings also illustrate that several disease-related factors such as dietary changes, obesity and insulin resistance can work in tandem to elevate disease risk in indigenous peoples undergoing rapid transitions.

\section{Acknowledgements}

The present study was supported by the Niskamoon Corporation. The authors thank the community research staff and participants. The authors' responsibilities were as follows: Y. E. Z. analysed the data and drafted the manuscript; S. K. supervised the manuscript drafting; E. D. designed the study; P. J. carried out the fatty acid analysis; G. M. E. designed the study and supervised the data analysis. All authors contributed to the revision of the manuscript.

None of the authors have any financial or personal conflict of interest to disclose.

\section{References}

1. Olshansky SJ, Passaro DJ, Hershow RC, et al. (2005) A potential decline in life expectancy in the United States in the 21st century. $N$ Engl J Med 352, 1138-1145.

2. Ronti T, Lupattelli G \& Mannarino E (2006) The endocrine function of adipose tissue: an update. Clin Endocrinol 64, $355-365$.

3. Torrejon C, Jung UJ \& Deckelbaum RJ (2007) n-3 Fatty acids and cardiovascular disease: actions and molecular mechanisms. Prostaglandins Leukot Essent Fatty Acids 77, 319-326.

4. Shils ME, Shike M, Ross AC, et al. (2006) Modern Nutrition in Health and Disease, 10th ed. Philadelphia: Lippincott Williams \& Wilkins.

5. Harris WS, Miller M, Tighe AP, et al. (2008) Omega-3 fatty acids and coronary heart disease risk: clinical and mechanistic perspectives. Atherosclerosis 197, 12-24.

6. Russo GL (2009) Dietary $n-6$ and $n-3$ polyunsaturated fatty acids: from biochemistry to clinical implications in cardiovascular prevention. Biochem Pharmacol 77, 937-946.

7. Jung UJ, Torrejon C, Tighe AP, et al. (2008) n-3 Fatty acids and cardiovascular disease: mechanisms underlying beneficial effects. Am J Clin Nutr 87, 2003S-2009S.

8. Warensjo E, Ohrvall M \& Vessby B (2006) Fatty acid composition and estimated desaturase activities are associated with obesity and lifestyle variables in men and women. Nutr Metab Cardiovasc Dis 16, 128-136.

9. Warensjo E, Riserus U \& Vessby B (2005) Fatty acid composition of serum lipids predicts the development of the metabolic syndrome in men. Diabetologia 48, 1999-2005.

10. el Boustani S, Causse JE, Descomps B, et al. (1989) Direct in vivo characterization of $\Delta 5$ desaturase activity in humans by deuterium labeling: effect of insulin. Metabolism 38, 315-321.

11. Rodríguez $\mathrm{Y} \&$ Christophe $\mathrm{AB}$ (2005) Long-chain $\omega 6$ polyunsaturated fatty acids in erythrocyte phospholipids are associated with insulin resistance in non-obese type 2 diabetics. Clinica Chimica Acta 354, 195-199.

12. Pan DA, Lillioja S, Milner MR, et al. (1995) Skeletal muscle membrane lipid composition is related to adiposity and insulin action. J Clin Invest 96, 2802-2808.

13. Young TK, Moffatt ME \& O'Neil JD (1993) Cardiovascular diseases in a Canadian Arctic population. Am J Public Health 83, 881-887.

14. Blanchet C, Dewailly E, Ayotte P, et al. (2000) Contribution of selected traditional and market foods to the diet of Nunavik Inuit women. Can J Diet Pract Res 61, 50-59.

15. Health Canada (2007) Nutrient value of some common foods, fish and shellfish. http://www.hc-sc.gc.ca/fn-an/nutrition/ fiche-nutri-data/nutrient_value-valeurs_nutritives-table8-eng.php (accessed 8 June 2008).

16. United States Department of Agriculture (2008) What's in the foods you eat search tool, 3.0. http://www.ars.usda.gov/ Services/docs.htm?docid=17032 (accessed 8 June 2008). 
17. Dewailly E, Blanchet C, Gingras S, et al. (2001) Relations between $n-3$ fatty acid status and cardiovascular disease risk factors among Quebecers. Am J Clin Nutr 74, 603-611.

18. Robinson E (1988) The health of the James Bay Cree. Can Fam Physician 34, 1609-1613.

19. Brassard P, Robinson E \& Lavallee C (1993) Prevalence of diabetes mellitus among the James Bay Cree of northern Quebec. CMAJ 149, 303-307.

20. Dewailly E, Blanchet C, Gingras S, et al. (2002) Cardiovascular disease risk factors and $n-3$ fatty acid status in the adult population of James Bay Cree. Am J Clin Nutr 76, 85-92.

21. Blond JP, Henchiri C \& Bezard J (1989) $\Delta 6$ and $\Delta 5$ Desaturase activities in liver from obese Zucker rats at different ages. Lipids 24, 389-395.

22. Egeland GM, Denomme D, Lejeune P, et al. (2008) Concurrent validity of the International Physical Activity Questionnaire (IPAQ) in an liyiyiu Aschii (Cree) community. Can J Public Health 99, 307-310.

23. Health Canada (2003) Canadian guidelines for body weight classification in adults - quick reference tool for professionals. http://www.hc-sc.gc.ca/fn-an/nutrition/weights-poids/ guide-ld- adult/cg_quick_ref-ldc_rapide_ref_e.html (accessed 11 April 2008).

24. Shaikh NA \& Downar E (1981) Time course of changes in porcine myocardial phospholipid levels during ischemia. A reassessment of the lysolipid hypothesis. Circ Res 49, $316-325$.

25. Lepage G \& Roy CC (1986) Direct transesterification of all classes of lipids in a one-step reaction. J Lipid Res 27, $114-120$

26. Canadian Diabetes Association (2003) Clinical Practice Guidelines for the Prevention and Management of Diabetes in Canada, Part 2: Type 2 Diabetes Mellitus. www.canadianguidelines.com/PDF/14_Diabetes_Type_II.pdf (accessed 12 December 2008).

27. Kuhnlein HV, Receveur O, Soueida R, et al. (2004) Arctic indigenous peoples experience the nutrition transition with changing dietary patterns and obesity. J Nutr 134, 1447-1453.

28. Appavoo D, Kubow S \& Kuhnlein H (1991) Lipid composition of indigenous foods eaten by the Sahtu (Hareskin) Dene-Metis of the northwest territories. J Food Comp Anal 4, 107-119.

29. Kuhnlein HV, Kubow S \& Soueida R (1991) Lipid components of tranditional Inuit foods and diets of Baffin Island. $J$ Food Comp Anal 4, 227-236.
30. Khan F, Elherik K, Bolton-Smith C, et al. (2003) The effects of dietary fatty acid supplementation on endothelial function and vascular tone in healthy subjects. Cardiovasc Res 59, 955-962.

31. Escudero A, Montilla JC, Garcia JM, et al. (1998) Effect of dietary $(n-9),(n-6)$ and $(n-3)$ fatty acids on membrane lipid composition and morphology of rat erythrocytes. Biochim Biophys Acta 2, 65-73.

32. Plourde M \& Cunnane SC (2007) Extremely limited synthesis of long chain polyunsaturates in adults: implications for their dietary essentiality and use as supplements. Appl Physiol Nutr Metab 32, 619-634.

33. Brown AJ, Roberts DC, Pritchard JE, et al. (1990) A mixed Australian fish diet and fish-oil supplementation: impact on the plasma lipid profile of healthy men. Am J Clin Nutr 52, 825-833.

34. Kuriki K, Nagaya T, Tokudome Y, et al. (2003) Plasma concentrations of $(n-3)$ highly unsaturated fatty acids are good biomarkers of relative dietary fatty acid intakes: a cross-sectional study. J Nutr 133, 3643-3650.

35. Baylin A, Kim MK, Donovan-Palmer A, et al. (2005) Fasting whole blood as a biomarker of essential fatty acid intake in epidemiologic studies: comparison with adipose tissue and plasma. Am J Epidemiol 162, 373-381.

36. Health Canada (2007) Map of Obesity According to Measured Body Mass Index (BMI) in Adults in Canada (both males and females). http://www.hc-sc.gc.ca/fn-an/surveill/atlas/map-carte/ mass_adult_obes_mf-hf_e.html (accessed 2 June 2008).

37. Centers for Disease Control (2005) Early release of selected estimates based on data from the 2004 National Health Interview Survey. www.cdc.gov/nchs/data/nhis/earlyrelease/200506_06.pdf (accessed 2 June 2008).

38. Decsi T, Molnar D \& Koletzko B (1996) Long-chain polyunsaturated fatty acids in plasma lipids of obese children. Lipids $\mathbf{3 1}$, 305-311.

39. Cunnane SC, Manku MS \& Horrobin DF (1985) Abnormal essential fatty acid composition of tissue lipids in genetically diabetic mice is partially corrected by dietary linoleic and $\gamma$-linolenic acids. Br J Nutr 53, 449-458.

40. Hughes S \& York DA (1985) Hepatic $\Delta 6$-desaturase activity in lean and genetically obese ob/ob mice. Biochem J 225, 307-313.

41. Maniongui C, Blond JP, Ullmann L et al. (1993) Age-related changes in $\triangle 6$ and $\triangle 5$ desaturase activities in rat liver microsomes. Lipids 28, 291-297.

42. Takahashi R \& Horrobin DF (1988) Linoleic acid-induced fatty acid changes in platelet and aorta of the rat: effect of age and cholesterol. Lipids 23, 885-890. 